\title{
Possible Effect of Antibiotic-Supplemented Feed and Environment on the Occurrence of Multiple Antibiotic Resistant Escherichia coli in Chickens
}

\begin{abstract}
The purpose of this study was to determine the occurrence of antibiotic resistant Escherichia coli isolated from chicks and chickens. This study was carried out on three flocks of birds fed commercial feeds supplemented with antibiotics from three commercial farms. The chicks and chickens in the fourth flock were reared in a chicken house, given feed without antibiotic supplementation. Cloacal swabs were taken from 50 birds per flock at 1, 21 and 42-day old. A total of $507 \mathrm{E}$. coli were isolated from these birds. The resistance of E. coli isolated form 1day-old chicks to chloramphenicol $(10 \mu \mathrm{g})$, cephalothin $(30 \mu \mathrm{g})$, cephalaxin $(30 \mu \mathrm{g})$, enrofloxacin $(5 \mu \mathrm{g})$ and neomycin $(30 \mu \mathrm{g})$ was $0-45 \%$ compared to the other four antibiotics, nalidixic acid $(30 \mu \mathrm{g})$, streptomycin $(10 \mu \mathrm{g})$, tetracycline $(30 \mu \mathrm{g})$ and trimethoprim $(5 \mu \mathrm{g})$ which was $75-100 \%$. The rates of resistance to antibiotics increased with the age of the chicks. Most of the isolates were resistant to at least 6 to 7 antibiotics. The highest rates of resistance to antibiotics were seen in 21 and 42 day old chickens. Escherichia coli, Klebsiella and Pseudomonas sp. isolated from feed samples were resistant to 4-9 antibiotics. The study suggests that the colonization antibiotic-resistant E. coli in the intestinal tracts of chicks and chickens were not necessarily due to the use of antibiotics in the feed as supplementation but may also be acquired from the immediate "contaminated" environment.
\end{abstract}

Keyword: Antibiotic, Escherichia coli, Chickens 